\title{
Correlation Between Disease Stage and the Presence of Viable Circulating Tumor Cells in Endometrial Cancer
}

\author{
IMRICH KISS ${ }^{1,2}$, KATARINA KOLOSTOVA ${ }^{1}$, RAFAŁ MATKOWSKI ${ }^{3}$, MARCIN JEDRYKA $^{3}$, \\ ANDRZEJ CZEKAŃSKI ${ }^{3}$, JIRI PAVLASEK ${ }^{4}$ and VLADIMIR BOBEK ${ }^{1,5,6,7,8}$ \\ ${ }^{1}$ Department of Laboratory Genetics, University Hospital Kralovske Vinohrady, Prague, Czech Republic; \\ ${ }^{2}$ Department of Gynecology, Military University Hospital and \\ $3^{\text {rd }}$ Faculty of Medicine Charles University, Prague, Czech Republic; \\ ${ }^{3}$ Division of Surgical Oncology, Gynaecological Oncology and Department of Oncology, \\ Lower Silesian Cancer Center and Wroclaw Medical University, Wroclaw, Poland; \\ ${ }^{4}$ Department of Obstetrics and Gynecology, Hospital Liberec, Liberec, Czech Republic; \\ ${ }^{5} 3$ rd Department of Surgery University Hospital Motol and 1st Faculty \\ of Medicine Charles University, Prague, Czech Republic; \\ ${ }^{6}$ Department of Thoracic Surgery, Masaryk's Hospital, Usti nad Labem, Czech Republic; \\ ${ }^{7}$ Department of Histology and Embryology, Wroclaw Medical University, Wroclaw, Poland; \\ ${ }^{8}$ Department of Thoracic Surgery, Lower Silesian Cancer Center, Wroclaw, Poland
}

\begin{abstract}
Background/Aim: The presence of circulating tumor cells (CTCs) in the peripheral blood of patients with solid tumors is associated with a poor prognosis. However, there are limited data concerning the detection of CTCs in endometrial cancer (EC). The aim of this study was to evaluate the presence of CTCs in the peripheral blood of patients with EC. Materials and Methods: Peripheral blood samples from 92 patients who underwent a surgical procedure were evaluated using MetaCell ${ }^{\circledR}$ separation technology for CTCs. Results: CTCs were detected in 69 (75\%) patients with EC. Conclusion: CTCs were detected in a higher percentage of patients than in other studies. The results showed that the technology applied in this study can efficiently capture viable tumor cells in the blood that can be cultured while maintaining their original phenotype. This paper discusses the first successful culturing of human circulating endometrial cancer cells for further downstream functional and molecular characterization.
\end{abstract}

Endometrial cancer (EC), ovarian cancer and cervical carcinoma are the most common gynecological cancers. EC is a treatable cancer with a good prognosis because in $75 \%$

Correspondence to: Prof. Vladimir Bobek, MD, Ph.D., Department of Laboratory Genetics, University Hospital, Kralovske Vinohrady, Srobarova 50, 10034 Prague, Czech Republic. Tel: +420 26716 3578, e-mail: vbobek@centrum.cz

Key Words: CTCs, endometrial cancer, circulating tumor cells, culturing. of women the disease is confined to the uterus, while women with metastatic disease have between 7 and 12 months median survival (1). Therefore, EC requires a more effective individualized therapy at a cellular and molecular level.

One way to better understand the invasion and metastatic process in cancer is to isolate and analyze circulating tumor cells (CTCs). CTCs are tumor cells present in the circulatory system of patients with solid tumors. Detection and quantification of CTCs provide additional information on the stage of cancer and response to therapy. CTCs' molecular characterization offers a possibility to control better the metastatic process.

The presence of CTCs in the blood of patients and their clinical correlation has been described in various cancers (25). Regarding gynecological cancers, most reports on CTC research are related to ovarian cancer patients (6). Currently, there is scarcity of information about CTCs in patients with EC. The aim of this study, was to capture viable CTCs, culture them in vitro and compare the ability of captured cells to grow in vitro at different disease stages.

\section{Materials and Methods}

Patients. A total of 92 patients diagnosed with EC have been enrolled in the study in accordance with the Declaration of Helsinki. The study was approved by the Ethical Committee of Wroclaw Medical University, Wrocław, Poland (EK 800/2012). All patients signed the consent to be enrolled in the study. All patients were candidates for surgery treatment. Based on their informed consent, clinical data were collected from all participating patients. The patient characteristics are shown in Table I and Figures 1-3. For each patient, approximately 
Table I. Patient characteristics.

\begin{tabular}{|c|c|c|c|c|c|}
\hline & $\mathrm{n}$ & $\mathrm{CTC}+$ & CTC- & $\%$ & $p$-Value \\
\hline Total number of patients & 92 & 69 & 23 & 75 & \\
\hline \multicolumn{6}{|l|}{ Histology } \\
\hline Endometrial adenocarcinoma & 81 & 65 & 16 & 80 & \\
\hline Papillary serous adenocarcinoma & 9 & 5 & 4 & 56 & \\
\hline Endometrial stromal sarcoma & 1 & 0 & 1 & 0 & \\
\hline Clear cell adenocarcinoma & 1 & 1 & 0 & 100 & \\
\hline \multicolumn{6}{|l|}{ Grade } \\
\hline Grade 1 & 29 & 23 & 6 & 79 & 0.648 \\
\hline Grade 2 & 46 & 33 & 13 & 72 & \\
\hline Grade 3 & 16 & 13 & 3 & 81 & \\
\hline \multicolumn{6}{|l|}{ FIGO } \\
\hline IA & 49 & 37 & 12 & 76 & 0.966 \\
\hline IB & 22 & 15 & 7 & 68 & \\
\hline II & 13 & 10 & 3 & 77 & \\
\hline IIIA & 1 & 1 & 0 & 100 & \\
\hline IIIC1 & 3 & 3 & 0 & 100 & \\
\hline IIIC2 & 3 & 2 & 1 & 67 & \\
\hline \multicolumn{6}{|l|}{ Further staging } \\
\hline Lymph node involvment YES & 6 & 5 & 1 & 83 & 0.616 \\
\hline Lymph node involvment NO & 85 & 63 & 22 & 74 & \\
\hline Peritoneal carcinomatosis & 1 & 1 & 0 & 100 & N/A \\
\hline Ascites & 0 & 0 & 0 & $\mathrm{~N} / \mathrm{A}$ & N/A \\
\hline Residual disease & 0 & 0 & 0 & N/A & N/A \\
\hline
\end{tabular}

$8 \mathrm{ml}$ of venous blood was drawn from the antecubital veins and placed into S-Monovette tubes (Sarstedt AG \& Co., Numbrecht, Germany) containing $1.6 \mathrm{mg}$ EDTA/ml blood as an anticoagulant. The samples were processed at room temperature using an isolation procedure completed within 24 hours after blood draw.

CTCs enrichment and culture. A size-based separation method for viable CTC-enrichment from peripheral blood was used (MetaCell ${ }^{\circledR}$, MetaCell s.r.o., Ostrava, Czech Republic) (7-9). The size-based enrichment process is based on the filtration of peripheral blood through a porous polycarbonate membrane (pores of $8 \mu \mathrm{M}$ in diameter). The standard $8 \mathrm{ml}$ of peripheral blood from patients suffering with EC was transferred to the filtration tube. The peripheral blood flow is supported by capillary action of the absorbent touching the membrane filter. The captured CTCs were observed immediately after filtration on the membrane. CTC presence was controlled immediately after isolation steps to avoid false negative results of examination. The membrane filter, which is kept in a plastic ring, was transferred directly into a 6-well culture plate and $4 \mathrm{ml}$ RPMI media containing $10 \%$ FBS was added to the membrane top and CTCs were cultured on the membrane in vitro under standard cell culture conditions $\left(37^{\circ} \mathrm{C}, 5 \% \mathrm{CO}_{2}\right.$ atmosphere) for a period of minimum 14 days on the membrane. The cultured cells were analyzed by histochemistry (May-Grünwald staining) and unspecific DAPI staining (Sigma, Munich, Germany) (Figure 4). Alternatively, the enriched CTC fraction was transferred from the membrane and cultured directly on any plastic surface or microscopic slide. Microscopic slide culture was preferred if immunohistochemistry/immunofluorescence analysis was planned. If an intermediate CTC analysis was awaited, the CTC fraction was transferred in PBS $(1.5 \mathrm{ml})$ to a cytospin slide. The slide was then dried for $24 \mathrm{~h}$ and analyzed immunohistochemically.
Cytomorphological analysis. The cells fixed and stained on the membrane were examined using light microscopy in two steps: (i) screening at $\times 20$ magnification to locate the cells and (ii) observation at $\times 40$ magnification for detailed cytomorphological analysis. Isolated cells and/or clusters of cells of interest (immunostained or not) were selected, digitized and examined by an experienced researcher and/or pathologist. CTCs were defined as cells presenting the following characteristics: (i) cell size equal or larger than $15 \mu \mathrm{m}$; (ii) nuclear size equal to or larger than $10 \mu \mathrm{m}$ ); (iii) irregularity of the nuclear contour; (iv) presence of a visible cytoplasm; (v) prominent nucleoli; (vi) high nuclear-cytoplasmic ratio; (vii) cluster presence; (viii) mitosis presence.

Statistical analysis. All analyses were performed using clinicopathological information transformed into variables 0 and 1 if applicable for tested characteristics. Chi-squared test, $t$-tests, cluster analysis and correlation analysis were performed using GeneX (MultiD, SE) and GraphPadPrism vs. 5 (Graphpad, US). p-Value of less than 0.05 was considered statistically significant.

\section{Results}

In our study, 92 patients with EC were examined for circulating tumor cells (CTCs). Patient characteristics are presented in Table I. CTCs were successfully isolated in 69 out of 92 patients ( $75 \%$ positivity).

Here, a successful isolation method of CTCs with proliferation potential in patients with EC is described. The cells captured by a size-based filtration method showed a viable character. The viability of CTCs was only minimally 


\section{Distribution of patients by histology}

11

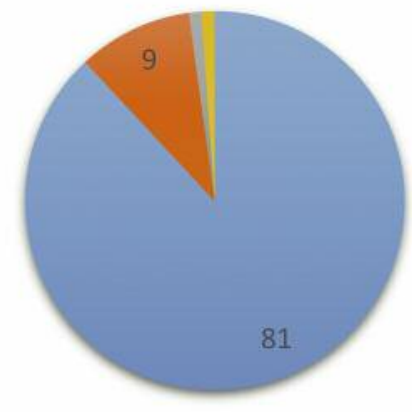

Endometrial adenocarcinoma

Endometrial stromal sarcoma

- Papillary serous adenocarcinoma

w Clear cell adenocarcinoma

Figure 1. Distribution of patients by histology.

\section{Distribution of patients by grading}

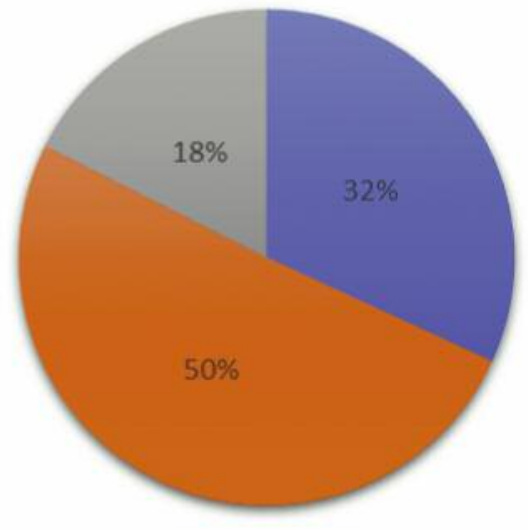

- Grade 1 arade 2 arade 3

Figure 2. Distribution of patients by grading.

affected by the isolation procedure and completely unaffected by the used cultivation media or bounded antibodies. CTCs were cultured in vitro and were grown in vitro in several cases for as long as 6 months as a standard cell culture (Figure 4).

The distribution of EC histological subtypes and CTC detection is shown in Table I and Figures 5-7. The majority of patients $(n=81)$ had endometrial adenocarcinoma with $80 \%$ CTC positivity, whereas the group of patients with papillary serous adenocarcinoma $(n=9)$ had only $56 \%$ positivity. No CTCs were found in the sample with endometrial stromal

\section{Distribution of patients by FIGO staging}

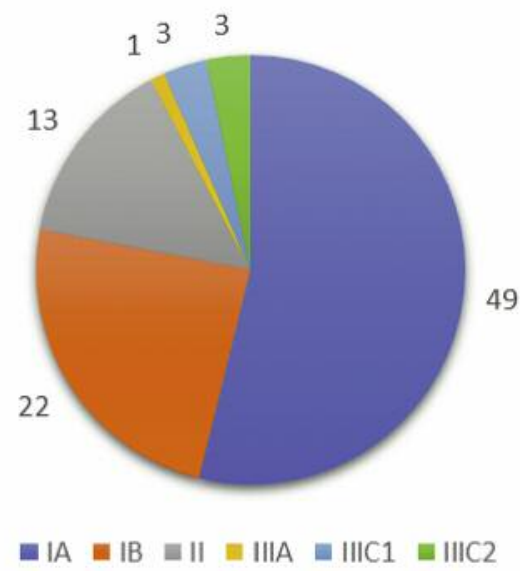

Figure 3. Distribution of patients by FIGO stating.
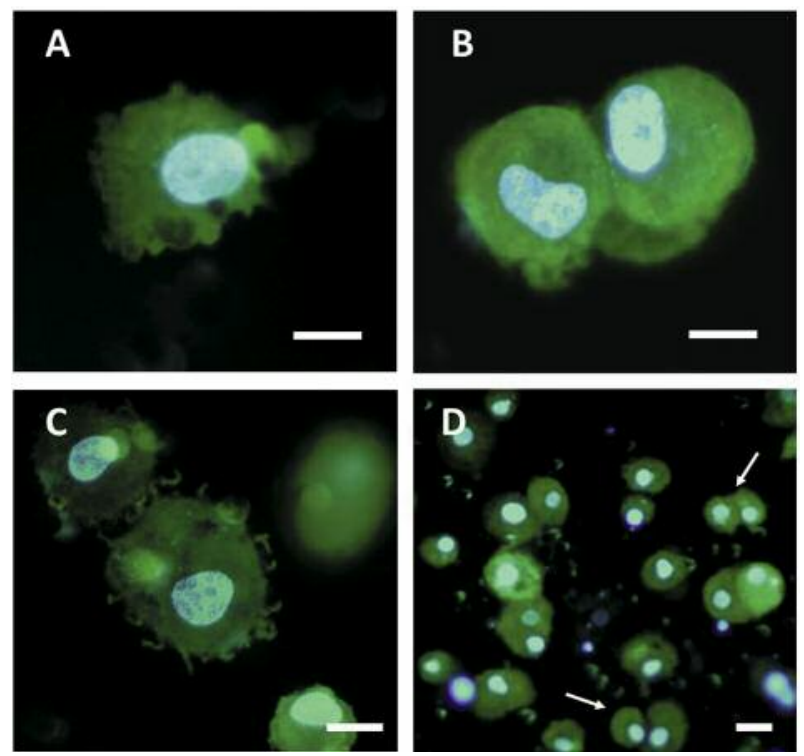

Figure 4. Isolated CTCs of endometrial cancer visualized by vital fluorescent staining. The arrows indicate mitosis presence. Bars represent 10 microns.

carcinoma $(n=1)$, CTCs were detected in the peripheral blood of a patient with clear cell adenocarcinoma $(n=1)$. Characterization by disease grade and CTC detection is shown in Figure 6. There was no significant difference between CTC presence and differentiation level (grade) of the cancer $(p=0.648)$. The stratification of patients into stages by FIGO is presented in Figure 7 . There is no correlation between the stage of the disease and CTC positivity $(p=0.966)$ or lymph node involvement and CTC detection $(p=0.616)$. Captured CTCs grow independently of disease stage. All these results 
CTC positivity by histology (\%)

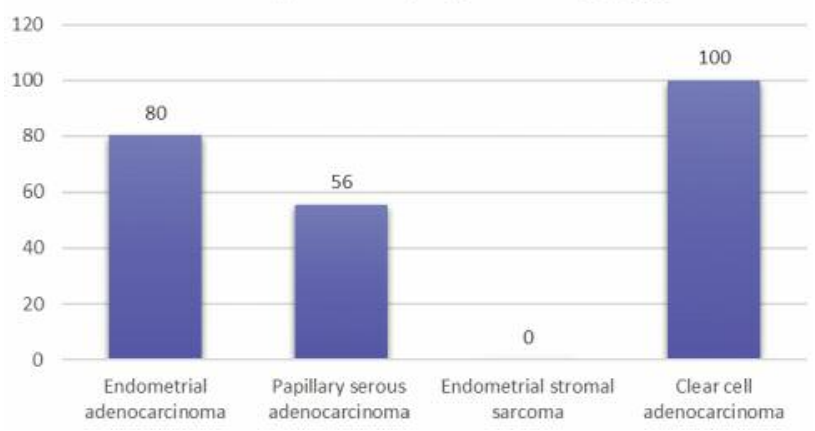

Figure 5. CTC positivity by histology.

indicate that the detection of CTCs in the peripheral blood is independent of the grade and stage of the cancer and of lymph node involvement.

\section{Discussion}

The aim of this study was to isolate viable CTCs from the peripheral blood of patients with $\mathrm{EC}$, culture them in vitro and compare the cultured cells with disease stage. Only limited data are presented regarding the presence of CTCs or disseminated tumor cells (DTCs) in EC patients. DTCs in the bone marrow of patients were observed using immunocytochemistry in $17 \%$ of patients with EC (10). RTPCR method was used in the first studies describing detection of CTCs in EC patients $(11,12)$. RT-PCR was used to determine the expression of cytokeratins (CK), namely CK19 or CK-20 in the peripheral blood. The results were very ambiguous and ranged from 10 to $51 \%$ positive patients.

Overall, 28 patients were included in the study that evaluated CTC presence in patients with high-risk endometrial cancer (13). Two of 28 (7\%) patients were positive for CTCs. The presence of positive CTCs was significantly associated with myometrial invasion and lymph node positivity. Only patients with endometrioid histology had positive CTCs. CTC detection rate was very low in this study. The authors used CellSearch technology which is dependent on the expression of epithelial cell adhesion molecule (EpCAM) by CTCs. It seems that using methods that are dependent on EpCAM can result in false negative detection of CTCs (14). This aspect was confirmed in another study which again used the CellSearch method (15). This study included 40 patients and only 15 percent of patients had one or more CTCs. EpCAM and cytokeratins are widely expressed in most epithelial malignancies but CTCs undergo epithelial-mesenchymal transition and during this process they lose expression of specific epithelial markers such as EpCAM (16).

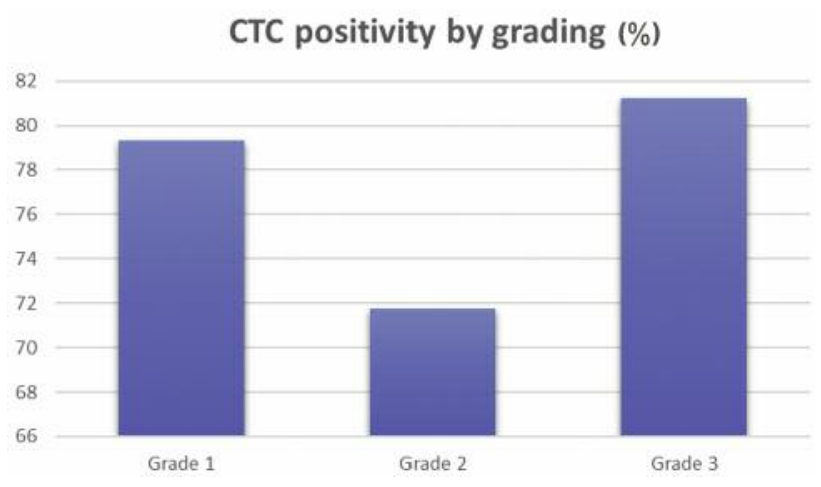

Figure 6. CTC positivity by grading.

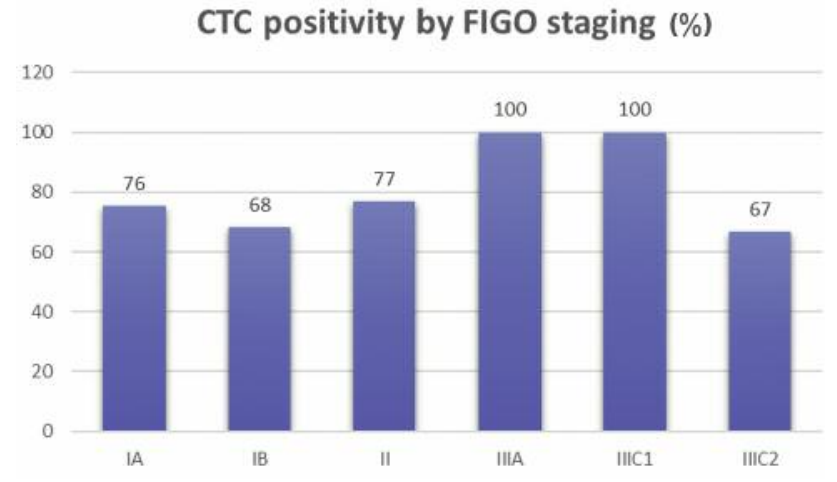

Figure 7. CTC positivity by FIGO stating.

In the reported study, a size-based method for separation of CTCs was used which is very efficient in detecting malignant cells with down-regulated epithelial markers. In the antigen-independent mode, the system can isolate CTCs from cancers that have lost or never had the epithelial marker characteristics. So, the fact that MetaCell ${ }^{\circledR}$ technology is independent of specific epithelial markers is the main reason for a higher detection of positive CTCs than that reported in the previous studies mentioned above.

Moreover, since the tumor cells are gently captured on the membrane, they may be used for further analysis at the cellular or molecular level. Separation of viable and intact CTCs provides a possibility of morphological investigation in addition to immunohistochemistry and RNA and/or DNA based PCR. We also believe that successful CTC cultures in vitro will provide important and necessary insight into the metastatic process.

Interesting results were obtained by comparing the presence of CTCs in patients who were subjected only to surgery and those who were given adjuvant radiotherapy. In the latter group the elimination of CTCs was significantly 
higher, but the patients had already undergone surgery which means that the primary site of cancer that disseminated cancer cells to the circulatory system had already been removed. Regarding the surgery group, the blood for examination was drawn before a radical surgery. We suggest that surgical eradication in EC significantly reduces the number of CTCs, which may be useful for follow-up since recurrence of CTCs might indicate cancer recurrence.

More information will surely be obtained in the future by correlating the presence of CTCs and disease-free survival (DFS) and 5-year overall survival (5-y-OS).

However, more studies concerning the role of CTCs in endometrial cancer patients are needed before this method is introduced into everyday clinical practice.

In conclusion, CTCs were present in $75 \%$ of patients with endometrial cancer. The high percentage of CTC positivity in the peripheral blood of patients with well differentiated tumors and early-stage carcinomas shows the metastatic potential of the disease. Moreover, CTCs show no correlation with the grade, stage or lymph node involvement and thus could be used as an independent diagnostic and treatment effectivity marker. The results show that the technology applied in this study can efficiently capture viable tumor cells from the blood which can then be cultured while maintaining the original phenotype. Viable CTCs can be efficiently isolated and in vitro cultures of endometrial cancer can be successfully established for downstream functional and molecular characterization.

\section{Conflicts of Interest}

VB and KK are inventors of the patent EP3008162B1 for the device used in this study for CTC separation.

\section{Acknowledgements}

This study was supported by the Minister of Science and Higher Education of Poland and performed as part of the subject registered in the Simple system at number ST.C280.17.010.

\section{References}

1 Oza AM, Elit L, Tsao MS, Kamel-Reid S, Biagi J, Provencher DM, Gotlieb WH, Hoskins PJ, Ghatage P, Tonkin KS, Mackay HJ, Mazurka J, Sederias J, Ivy P, Dancey JE and Eisenhauer EA: Phase II study of temsirolimus in women with recurrent or metastatic endometrial cancer: a trial of the NCIC Clinical Trials Group. J Clin Oncol 29(24): 3278-3285, 2011.

2 Bobek V, Gurlich R, Eliasova P and Kolostova K: Circulating tumor cells in pancreatic cancer patients: Enrichment and cultivation. World J Gastroenterol 20(45): 17163-17170, 2014.

3 Bobek V, Matkowski R, Gürlich R, Grabowski K, Szelachowska J, Lischke R, Schützner J, Harustiak T, Pazdro A, Rzechonek A and Kolostova K: Cultivation of circulating tumor cells in esophageal cancer. Folia Histochem Cytobiol 52(3): 171-177, 2014.
4 Eliasova P, Kolostova K, Kobierzycki C and Bobek V: Clinical studies monitoring circulating and disseminated tumor cells in gastrointestinal cancers. Folia Histochem Cytobiol 51(4): 265277, 2013.

5 Kolostova K, Matkowski M, Jędryka M, Soter K, Cegan M, Pinkas M, Pavlasek J, Spicka $\mathbf{J}$ and Bobek V: The added value of circulating tumor cells examination in ovarian cancer staging of ovarian cancer. Am J Cancer Res 5(11): 3363-3375, 2015.

6 Romero-Laorden N, Olmos D, Fehm T, Garcia-Donas J and Diaz-Padilla I: Circulating and disseminated tumor cells in ovarian cancer: a systematic review. Gynecol Oncol 133(3): 632639, 2014.

7 Kolostova K, Cegan M and Bobek V: Circulating tumor cells in patients with urothelial tumors: Enrichment and in vitro culture. CUAJ-Canadian Urological Association Journal, Can Urol Assoc J 8(9-10): E715-E720, 2014.

8 Kolostova $\mathrm{K}$, Zhang Y, Hoffman RM and Bobek V: In vitro culture and characterization of human lung cancer circulating tumor cells isolated by size exclusion from an orthotopic nudemouse model expressing red fluorescent protein. J Fluoresc 24(5): 1531-1536, 2014.

9 Kolostova K, Spicka J, Matkowski R and Bobek V: Isolation, primary culture, morphological and molecular characterization of circulating tumor cells in gynecological cancers. Am J Transl Res 7(7): 1203-1213, 2015.

10 Fehm T, Becker S, Bachmann C, Beck V, Gebauer G, Banys M, Wallwiener D and Solomayer EF: Detection of disseminated tumor cells in patients with gynecological cancers. Gynecol Oncol 103(3): 942-947, 2006.

11 Klein A, Fishman A, Zemer R, Zimlichman S and Altaras MM: Detection of tumor circulating cells by cytokeratin 20 in the blood of patients with endometrial carcinoma. Gynecol Oncol 78(3): 352-355, 2000.

12 Ji XQ, Sato H, Tanaka H, Konishi Y, Fujimoto T, Takahashi O and Tanaka T: Realtime quantitative RT-PCR detection of disseminated endometrial tumor cells in peripheral blood and lymph nodes using the LightCycler System. Gynecol Oncol 100(2): 355-360, 2006.

13 Bogani G, Liu MC, Dowdy SC, Cliby WA, Kerr SE, Kalli KR, Kipp BR, Halling KC, Campion MB and Mariani A: Detection of circulating tumor cells in high-risk endometrial cancer. Anticancer Res 35(2): 683-687, 2015.

14 Lin H, Balic M, Zheng S, Datar R and Cote RJ: Disseminated and circulating tumor cells: Role in effective cancer management. Crit Rev Oncol Hematol 77(1): 1-11, 2011.

15 Ni T, Sun X, Shan B, Wang J, Liu Y, Gu SL and Wang YD: Detection of circulating tumour cells may add value in endometrial cancer management. Eur J Obstet Gynecol Reprod Biol 207: 1-4, 2016.

16 Friedlander TW, Premasekharan G and Paris PL: Looking back, to the future of circulating tumor cells. Pharmacol Ther 142(3): 271-280, 2014. 